\title{
Notes on the Phonology of Southern Kurmanji
}

\author{
BY E. B. SOANE
}

THE allocation of its proper place among Iranian languages to Kurmanji (Kurdish) has been rendered well-nigh impossible up to the present by the lack of material available for examination. The most careful Orientalists tend to consider it a non-Persian language, notably Justi, Darmesteter, and Socin (in the Grundriss der Iranischen Philologie). The first emphatically states that it is in no way a degraded New Persian, and that a description of the peculiarities of Iranian speech would not be exhaustive if the phonetics and etymology of Kurdish be disregarded. ${ }^{1}$

The second, confronted with many apparent phonological incongruities, leaves it an open question, but places importance upon the preservation of the Avestic $z$, which both in Old Persian and New Persian is represented by $d$ in certain conditions. Examples of this will be seen in the notes below.

Darmesteter furthermore lays stress upon the necessity of studying the material of a single dialect in order to avoid confusion. I have carefully excluded from the following notes conzideration of any but the great southern section of Kurmanji.

As bearing upon the apparent incongruities mentioned above I would quote a speculation of Darmesteter which study of the language has now proved to me to have been of the greatest importance. He says: "Ici se pose la question inextricable des emprunts au persan: l'identité de forme entre un mot persan et un mot kurde est, en général, un indice que le mot kurde est emprunté." 2

1 Justi, Introduction to Kurdische Grammatik.

${ }^{2}$ Darmesteter, Etudes Iraniennes, vol. ii, p. 89. 
In the section on Kurdish in the Grundriss der Iranischen Philologie, Socin prefaces his general remarks with the statement that Kurdish does not stand either to Pehlevi or New Persian in the relation of "sister-dialect", but that there is something remoter in the relationship. He further considered that Kurdish does not come from Old Persian, and advised the comparison of the former with New Persian, in order to determine in which particulars it exhibits older or younger word-forms.

I hope that the following notes may throw some new light upon the subject, and serve as a preliminary step to further investigation of a widely-spoken language which has hitherto suffered neglect because of our ignorance of it.

I venture to think that sufficient individuality will be seen to invite further research, for though, as might be expected, Kurmanji is in consonance with Pablevi and New Persian in many of its differences and developments from Avestic, it has quite enough characteristics of its own to render its description as a dialect of one of the two younger languages very hazardous.

Of its well-preserved grammar there is not space to treat here, nor of its peculiar affinities with Pushto, Baluchi, and Ossethian.

I am aware that I have not done full justice to the vowel sounds, which require further subdivision, but the subject is a very large one, which demands separate treatment.

Abbreviations used are :-

$$
\begin{array}{ll}
\text { Av. }=\text { Avestic. } & \text { Phl. = Pehlevi. } \\
\text { NP. = New Persian. } & \text { Slkt. = Sanskrit. } \\
\text { Tk. = Turkish. } & \text { Ar. = Arabic. } \\
\text { Kj. = Kurmanji. } & \text { OP. = Old Persian. }
\end{array}
$$

\section{VOWELS}

Short : a $e$ io $u$.

Long : $\bar{a} \overline{\bar{s}} \bar{\imath} \bar{o} \bar{a} \underline{u}$. 


\section{SHORT $a$}

1. Kj. $a=$ Av. $a$, Phl. $a$, NP. $a$.

wafr, snow: Av. vafra, Phl. vafr, NP. barf. pas, small cattle: Av. pasu-, Phl. pah, NP. - wan, a common mountain tree: Av. vanā, Phl. van, NP. - hakar, aka, hagar, if : Av. ha-kara, Phl., NP. agar. hamār, a store-room : Av. ham $\sqrt{b e r e, ~ P h l ., ~ N P . ~ a m b a ̄ r . ~ a n g u s t, ~ f i n g e r: ~ A v . ~}$ angushta, Phl. angust, NP. angusht. awa, that: Av. ava, Phl. ava, NP. - han-, verbal prefix: Av. han-, Phl., NP. an-. warāz, boar: Av. varāza-, Phl. varāz, NP. gurāz. asr, tears : Av. asru, Phl., NP. ars. az, aze, I : Av. azem, Phl., NP. - .

2. Kj. $a=$ Av. $a$, Phl. $\bar{a}$, NP. $\bar{a}$.

bar, loose, free: Av. fra-, Phl. frāj, NP. firāz. man, appertaining to: Av. manah-, Phl., NP. -mān. awand, that so much : Av. avavant, Phl. hãvant, NP. -.

3. Kj. $a=$ Av. $a \bar{e}$, Phl. $i$, NP. $i$.

$a m$, this: Av. aèm, Phl. ima, NP. im-.

4. Kj. $a=$ Av. $a i$, Phl. $a$, NP. $a$.

gar, mountain : Av. gairi-, Phl., NP. -. kan̄, maiden : Av. kaine, Phl. kanīk, NP. kanī $[z$.

5. Kj. $a=$ Av. $\bar{a}$, Phl. $\bar{a}, \mathrm{NP} . \bar{a}$.

$a$, to: Av. $\bar{a}, \mathrm{Phl} . \bar{a}, \mathrm{NP} .-\sqrt{ }$ aparmū, trust: Av. $\bar{a}-\sqrt{ }$ parmū, Phl., NP. - danik, a pip: Av. dāno-, Phl. dānak, NP. dānah. ga, time, place: Av. gātu-, Phl. gās, NP. gāh. pata, a pen for animals: Av. pāta-, Phl., NP. -

\section{INONG $\bar{a}$}

Pronunciation very much lighter than the NP. $\bar{a}$, practically the same as in English "half", "father".

1. $\mathrm{Kj} . \bar{a}=\mathrm{Av} . \bar{a}, \mathrm{Phl} . \bar{a}, \mathrm{NP} . \bar{a}$.

$\bar{a} s$-, gazelle: Av. āsu-, Phl. $\bar{a} h \bar{u} k$, NP. $\bar{a} h \bar{u} . \quad m \bar{a} \underline{l}$, nouse, family: Av. nmāna-, Phl. mān, NP. mān. pānīa, heel : Av. päshna-, Phl. pāshnak, NP. pāshna. āst, alignment: Av. rästa-, Phl. rāst, NP. rāsta. āgir, fire: Av. ätar-, Phl JRAS. APRIL 1922. 
$\bar{a} t \bar{a}$, NP. ā $\delta a r . \quad b r a \bar{a}$, brother: Av. brātar-, Phl. brāt, NP. barādar. wā, wind: Av. vāta-, Phl. vāt, NP. bād. hāwīn,

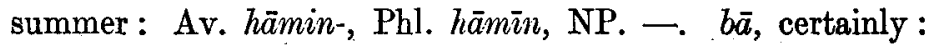
Av. $b \bar{a}-$, Phl., NP. - $k \bar{a} r$, relatives, people: Av. kāra-, Phl., NP. -. $\sqrt{ } \bar{a} z h u$, drive, urge: Av. $\bar{a} \sqrt{ } z i, a z$, Phl., NP. - -

2. Kj. $\bar{a}=\mathrm{Av} . a$, Phl. -, NP. 一.

$\bar{a} w \bar{a}$, down: Av. ava (seen in $\mathrm{Phl}$. and NP. only as prefixial $\bar{o}$ to verbal roots). $\sqrt{ } n \bar{a} s h$, become interred: Av. $\sqrt{ }$ nash.

2a. Kj. $\bar{a}=$ Av. $a$, Phl. $a$, NP. -

hawār, an encampment or camping ground: Av. vara , Phl. var, NP. -

2b. Kj. $\bar{a}=$ Av. $a$, Phl. $a$, NP. $a$.

pāsh, after, behind: Av. pascha, Phl., NP. pas. sār, cold : Av. sareta-, Phl. sart, NP. sard.

3. Kj. $\bar{a}=$ Av. $a$, Phl. $\bar{a}, \mathrm{NP} . \bar{a}$.

jūān, young: Av. yuvan-, Phl. yovān, NP. javān. kānī, a spring: Av. khan-, Phl. khānik, NP. khānī. ferā, broad : Av. fratah, Phl. frāh, NP. firākh. hazhār, miserable: Av. $\sqrt{ }$ zar, Phl. zhār, NP. zār. māsì, fish : Av. masya-, Phl. $m \bar{a} h i k$, NP. $m \bar{a} h \bar{\imath}$.

4. Kj. $\bar{a}=\mathrm{Av}, a \theta, \mathrm{Phl} . a h, \mathrm{NP} . a h$.

zhār, poison: Av. jatra, Phl., NP. zahr. pān, broad :

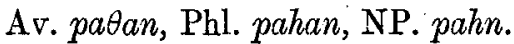

5. $\mathrm{Kj} \cdot \bar{a}=a h, a h$ in loan-words. jāsh, a donkey foal: Ar. jahhsh. kārēz, a conduit: NP. kāhriz.

\section{Short $e$.}

1. $\mathrm{Kj}, e=\mathrm{Av}$. , , Phl. $u$, NP. $u$.

dahem, tenth : Av. dasoma, Phl., NP. dahım. berz, high : Av. baraz, Phl., NP. burz. perd, bridge: Av. paratu-, Phl. puhr, puhl, NP. pul. 
2. Kj. $e=$ Av. $\bar{a} i$, Phl. $\bar{a} y$, NP. $\bar{a}, \bar{a} y$. $\sqrt{ } e$, come: Av. $\sqrt{ } \bar{a}-i, \mathrm{Phl} . \sqrt{ } \bar{a} y, \mathrm{NP} . \sqrt{ } \bar{a}, \bar{a} y$.

3. Kj. $e=$ Av. $a$, Phl. $a$, NP. $a, u$.

est, bone: Av. asta-, Phl. ast, NP. ust (in ustakhwan): nezīk, near : Av. nazda-, Phl., NP. nazd.

\section{LoNG $\bar{e}$}

Pure vowel sound as $\hat{e}$ in French béte, not ai diphthong.

1. Kj. $\tilde{e}=$ Av. $\bar{e}$, Phl. $\bar{e}$, NP. $\vec{e}$.

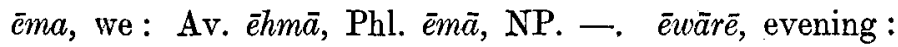
Av. —, Phl. èwārak, NP. èuārr.

2. $\mathrm{Kj} . \bar{e}=\mathrm{Av} . a i$, Phl. $a, \mathrm{NP} . a$.

pērī, fairy: Av. pairika-, Phl. parīk, NP. parī. pē, to: Av. paiti, Phl. pat-, NP. ba. kēeñ, girl: Av. kainē, Phl. kanīk, NP. kanī z.

3. Kj. $\bar{e}=\mathrm{A}$. $\vec{a} i$, Phl. $\bar{a} i, \mathrm{NP} . a$.

nèr, male: Av. näirya-, Phl. nāirik, NP. nar.

4. $\mathrm{Kj} . \bar{e}=\mathrm{Av} . a$, Phl. $a, \bar{a}$, NP. $a, \bar{a}$.

mèrg, turf: Av. mareghā-, Phl. -, NP. ? margh. pērē, yester: Av. parōayar, Phl. parēr, NP. parīr-. stēr, star : Av. star-,-Phl. star, NP. sitar. $\sqrt{ } b \bar{e} z h$, speak: Av. vach,Phl. vājj. NP. -. kērd, knife: Av. karata, Phl. kārt, NP. kārd. jēzhn, festival : Av. yasna-, Phl. yashn, NP. jashn. $k \bar{e} \underline{l}$, cultivation: Av. karsh-, Phl. kār, NP. kār. pē, foot: Av. pad-, Phl. pā̄, NP. pā. mēshik, brain : Av. mazga-, Phl. mazg, NP. maghz.

5. $\mathrm{Kj} . \bar{e}=\mathrm{Av} . a$, Phl. $\bar{\imath}, \mathrm{NP} . \bar{\imath}$.

$\sqrt{ } h \bar{e} l$, refrain, permit, leave alone: Av. $\sqrt{\text { hart, }}$ Phl., NP. $\sqrt{ } h \bar{\imath} l$ :

6. Kj. $\bar{e}=\mathrm{Av} . \bar{a}$, Phl. $\bar{a}, \mathrm{NP} . a, \bar{a}$.

drēzh, long: Av. drājo-, Phl. drāj, NP. dirāz. chēsht, a meal: Av. ?, Skt. $\sqrt{ }$ chāsh, Phl. chāsht, NP. chasht. $\sqrt{ } \bar{e} n \bar{a}, \sqrt{ } h \bar{e} n \bar{a}$, bring: Av. $\bar{a}-\sqrt{ } n a y$, Phl. -, NP. - 


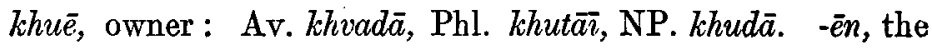
agent: Av. $\hat{\imath}, \mathrm{Phl}$, NP. $-\bar{a} n$.

6a. $\mathrm{Kj} \cdot \bar{e}=\bar{a}$ in loan-words.

kirē, hire, rent : Ar. kirā. ktēb, book : Ar. kitāb.

7. $\mathrm{Kj} . \bar{e}=\mathrm{Av} . a$, Phl. $\bar{o}, \mathrm{NP} . \bar{u}$.

pēst, skin, bark : Av. pasto-, Phl. pōst, NP. püst.

8. Kj. $\bar{e}=$ Av. $a e, a ə$, Phl. $\bar{e}, \mathrm{NP} . \bar{\imath}$.

bèn, nose: Av. vaəna-, Phl. vēnī, NP. bīnī. kēm, pus, mucus: Av. haam-, Phl. khēm, NP. khīm. $\bar{e}$, terminal indefinite article: Av. aeva, Phl. $\bar{e} v$, NP. $\bar{\imath}$. nēwa, vicinity : Av. naama, Phl. nemak, NP. --.

9. Kj. $\bar{e}=$ Av. ao, Phl. $\bar{a}$, NP. - .

$\sqrt{ } \bar{e} z h$, speak : Av. $\sqrt{ } a o j, a o g h z h$, Phl. $\sqrt{ } v a \tilde{j}$, NP. -

10. Kj. $\bar{e}=\mathrm{Av}$. ao (i), Phl. o, $\bar{o}, \mathrm{NP} . \bar{o}, \bar{u}$.

$h \bar{e} z, h \bar{e} z h$, strength : Av. aoja-, Phl. ōj, NP. - . bēn, smell : Av. baoidhi-, Phl. böd, NP. bō, bō̄. mèr, ant: Av. maoiri-, Phl. mōr, NP. mür.

11. Kj. $\bar{e}=$ Av. $a u$, Phl. $\bar{o}$, NP. $\bar{u}$.

kēf, mountain : Av. kaufa-, Phl. köf, NP. küh.

12. Kj. $\bar{e}=$ Av. $e$, Phl. $\bar{o}$, NP. $\bar{u}$.

rēwā, fox : Av. revish-, Phl. rōbōs, NP. rūbāh.

13. $\mathrm{Kj} . \bar{e}=\mathrm{Av} . i \theta$, Phl. $\bar{e} \delta, \mathrm{NP} .-$

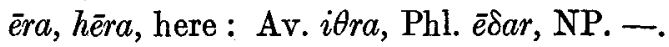

14. Kj. $\bar{e}=$ Av. ya, Phl. ya, NP. ja.

$\bar{e} s h$, pain, affliction : Av. yaska-, Phl. yask, NP. jask.

15. Kj. $\bar{e}=0$ in loan-word.

hēldāsh, travelling companion : Tk. yoldāsh.

V. SHORT $i$

1. Kj. $i=$ Av. $i$, Phl. $i$, NP. -

gisht, all : Av. vispo-, Phl. vist. nizm, low: Av. nitem-, nisma, Phl. -

2. Kj. $i=$ Av. $a$, Phl. $a$, NP. $a$. nim, dampness : Av. namna-, Phl., NP. nam. min, me: 
Av. mana, Phl., NP. man. kirdin, to do: Av. $\sqrt{ }$ kar, Phl. kartan, NP. kardan.

3. Kj. $i=$ Av. $a$, Phl. $a$, NP. $u$.

girō, gullet: Av. garah-, Phl. garūk, NP. gulū. $\sqrt{ } k i r$, gnaw : Av. $\sqrt{ }$ kar, Phl. $\sqrt{ }$ karīn, NP. -.

4. Kj. $i=$ Av. $a$, Phl. $u$, NP. $u$.

$\sqrt{ }$ bir, cut: Av. $\sqrt{ }$ bar, Phl., NP. $\sqrt{ }$ bur. $\sqrt{ }$ mir, die : Av. $\sqrt{ }$ mar, Phl., NP. $\sqrt{ }$ murd.

5. Kj. $i=$ Av. $a$, Phl. - , NP. - .

girj, wrinkle: Av. $\sqrt{ }$ garez, Phl., NP. -

6. Kj. $i=$ Av. a, Phl. $u$, NP. $u$.

mirishk, hen: Av. maragha-, Phl., NP. murgh. $\sqrt{ }$ pirs, ask : Av. $\sqrt{ }$ paras, Phl., NP. $\sqrt{ }$ purs.

7. Kj. $i=$ Av. aə, Phl. $\bar{e}, \mathrm{NP} . \bar{e}$.

mizh, mist : Av. mazgha-, Phl., NP. mēgh.

8. Kj. $i=$ Av. $u$, Phl. $u$, NP. $u$.

diz, thief : Av. duzhda-, Phl. duzhd, NP. duzd. dizhmin, enemy : Av. dushmainyu-, Pbl., NP. dushman. bin, bottom : Av. buna-, Phl., NP. bun. bizin, goat: Av. buja-, Phl. buj, NP. buz. mist, fist: Av. mushti-, Phl. must, musht, NP. musht. hishk, dry: Av. hushka-, Phl. khushka, NP. khushk. jigā, separate: Av. yuta-, Phl. jutak, NP. judā.

9. Kj. $i=$ Av. $a \bar{e}$, Phl. $\bar{e}, \mathrm{NP} . \bar{\imath}$. -in, adjectival suffix : Av. -aēna, Phl. -ēn, NP. -īn.

10. Kj. $i=$ Av. $i$, Phl. $i$, NP. $i$. $\sqrt{ }$ chin, pluck, cull : Av. $\sqrt{ }$ chinv, Phl. $\sqrt{ }$ chin, NP. $\sqrt{ }$ chinn.

\section{LoNG $\bar{\imath}$.}

1. Kj. $\bar{\imath}=$ Av. $\bar{\imath}$, Phl. $\bar{\imath}$, NP. $\bar{\imath}$.

bìr, reason, understanding: Av. vīra, Phl., NP. vīr. shīr, milk: Av. khshīra-, Phl., NP. shīr. $\sqrt{ } d \bar{r}$, see: Av. $\sqrt{ } d \bar{\imath}$, Phl., NP. $\sqrt{ } d \grave{\imath}$. 
2. $\mathrm{Kj} . \bar{\imath}=$ Av. $i$, Phl. $\bar{\imath}, \mathrm{NP} . \bar{\imath}$.

shīn, wailing: Av. khshim-, Phl. shīn, NP. 一. $\sqrt{ } z h \bar{\imath}$, live: Av. $\sqrt{ } j i v, \mathrm{Phl} . \sqrt{ } z \bar{i} v, \mathrm{NP}, \sqrt{ } z \bar{i}$.

3. Kj. $\bar{\imath}=$ Av. $i$, Phl. -, NP. $a$. bī̄anu, pretext: Av. vi- $\sqrt{ } d h \bar{a}$, Phl. vhānak, NP. bahāna.

4. $\mathrm{Kj} . \bar{\imath}=\mathrm{Av} . i$, Phl. $u, \mathrm{NP} . i$. ispi, louse: Av. spish, Phl, shapush, NP. shapish.

5. $\mathrm{Kj} . \bar{\imath}=\mathrm{Av} . a e, \mathrm{Phl} . \bar{\imath}, \bar{e}, \mathrm{NP} . \bar{\imath}$.

$s p \bar{\imath}$, white: Av. spaeta, Phl. spēt, NP. safīd. qūzh, locks : Av. gaesa-, Phl. gèe, NP. gìs. pìm, fat: Av. paem-, Phl. pim, NP. pi. shīn, blue, green: Av. khshaena-, Phl., NP. khashin. nīw, half : -Av. naema-, Phl., NP. nīm.

6. Kj. $\bar{\imath}=$ Av. $\bar{a}$, Phl. $\bar{a}$, NP. $\bar{a}$.

$\sqrt{ } s h \bar{\imath}$, being worthy: Av. $\sqrt{ } k h s h a \bar{y}$, Phl., NP. $\sqrt{ }$ shāy. shīw, evening meal : Av. khshäfniya, Phl., NP. shäm.

\section{SHORT o}

This vowel is not common, nor are there sufficient words of which the derivation is clear, for comparison. In the case of $o$ "and ", the equivalent in Av., Phl., and NP. is $u$, while in the word mort " myrtle", $u$ is seen in the NP. murad. In the word mokh " marrow of bones", it appears to represent Av. $a$ in mazga-, and Phl., NP. $a$ in mazg and maghz respectively. It occurs in the words nor "goggle-eyed", polk " pea, pod", rong, a kind of felt, the etymology of none of which is clear. The word tor "anger, chagrin, offence" appears to have a NP. counterpart in $t \bar{r}$. It also appears in the loan-words from Turkish, otrā $q$, qonāgh, komīte.

\section{LoNG $\bar{o}$}

1. $\mathrm{Kj} . \bar{o}=\mathrm{Av} . a$, Phl. ?, NP. $a$. dōl, a dale: Av. darena-, Phl. ?, NP. darra.

2. $\mathrm{Kj} . \bar{o}=\mathrm{Av}$. ae, Phl. ?, NP. $\bar{o}$. jōsh, white heat of iron: Av. $\sqrt{ } y a e s h$, Phl. ?, NP. jōsh. 
3. $\mathrm{Kj} . \bar{o}=\mathrm{Av} . \alpha o$, Phl. $\bar{o}, \mathrm{NP} . \bar{o}, \bar{u}$.

rō, day : Av. raochah-, Phl. rōch, NP. rōz, rūz. bōr, brown : Av. ?, Phl. ?; NP. būr. dōsh, a load for the back: Av. daosha-, Phl. dōsh, NP. düsh.

4. $\mathrm{Kj} . \bar{o}=\mathrm{Av}$. ava, Phl. ava, NP. aw.

ōra, there: Av. avaAra, Phl. -, NP. - nō, new : Av. nava, Phl. navak, NP. naw.

4a. Kj. $\bar{o}=$ Av. ? ava, Phl. $\bar{o}, \mathrm{NP} .-$.

$-\bar{o} k$, attributive suffix : Av. ? avaka-, Phl. $\bar{k} k$, NP. -.

5. Kj. $\bar{o}=\mathrm{Av} . a w$, Phl. ?, NP. $\vec{u}$.

gōza, a large jar : Av. kawza-, Pbl. ?, NP. kūza.

6. Kj. $\bar{o}=$ Av. $u$, Phl. $\bar{o}, \mathrm{NP} . \bar{o}$.

hōsh, reasoning: Av. ushi-, Phl., NP. hōsh. $\sqrt{ }$ dōsh, milk : Av. $\sqrt{ } d u z$, Phl., NP. $\sqrt{ } d \bar{s} s h$.

7. $\mathrm{Kj} . \bar{o}=$ Av. $u$, Phl. $a f$, NP. - .

gōra, large: Av. ugra-, Phl. gafra, NP. -

7a. Kj. $\bar{o}=u$ in loan-word.

$\sqrt{ }$ too $q$, bursting out: Ar.

8. $\mathrm{Kj} \cdot \bar{o}=u h$ in loan-word.

mōr, seal : NP. muhr.

9. Kj. $\bar{o}=$ Phl. $v a$, NP. $\bar{u}$ in loan-word.

khōsh, pleasant: Phl. khvash, NP. khīsh.

10. $\mathrm{Kj} . \bar{o}=\bar{o}$ in loan-words.

tōp, cannon : NP. tōp. tōz, dust: Tk. tōz.

IX. Short $u$

1. Kj. $u=$ Av. $u$, Phl. $u$, NP. $u$.

amust, finger: Av. angushta-, Phl. angust, NP. angusht. duwa, the rear, behind: Av. dum-, Phl. ?, NP. dum- (in dumbāl).

2. $\mathrm{Kj} \cdot u=$ Av. $u$, Phl. $u, \bar{u}$, NP. $\bar{u}$.

kutik, a fragment, small piece: Av. kutaka-, Phl. -, 
NP. -. zhuzhik, hedgehog : Av. duzhaka-, Phl. zhüzhak, NP. zhüzha.

3. Kj. $u=$ Av. $a$, Phl. $a$, NP. $a$.

$\sqrt{ }$ wush, sway, wave: Av. $\sqrt{ }$ vaza, Phl. $\sqrt{ }$ vaz, NP. qurna, corner, border: Av. karana-, Phl. kanārak, NP. kanār. mughägh, a low-lying place: Av. magha-, Phl. magh, NP. maghāk. pursha, sleet: Av. parshva-, Phl. parashveh, NP. - -

4. Kj. $u=$ Av. $a$, Phl. $a$, NP. $u$. qurg, throat, gullet : Av. garah-, Phl. garōk, galōk, NP. gulū.

5. Kj. $u=$ Av. $a o$, Phl. o, $u$, NP. $u, \bar{u}$.

dush, last night: Av. daosh-, Phl. dosh, NP. dūsh. $\sqrt{ } k u s h$, kill : Av. $\sqrt{ } k a o s h$, Phl. $\sqrt{ } k u s h$, NP. $\sqrt{ } k u s h$.

6. Kj. $u=$ Av. $\bar{u}$, Phl. $\bar{u}$, NP. $\bar{u}$.

$t u$, thou: Av. $t \bar{u}, t \bar{u} m$, Phl. $t \bar{u}$, NP. $t \bar{u}$.

7. Kj. $u=$ Av. e, Phl. $u$, NP. $u$.

gurchik, kidney: Av. veretka-, Phl. gurtak, NP. gurda. gurg, wolf: Av. vehrka-, Phl. gurg, NP. gurg.

8. Kj. $u=$ Av. $i$, Phl. -, NP. - . $\sqrt{ } n u s$, sleep : Av. $n i-\sqrt{ } s i$, Phl. - , NP. -

X. LoNG $\bar{u}$

1. Kj. $\bar{u}=$ Av. $\bar{u}$, Phl. $\bar{u}$, NP. $\bar{u}, u$.

$g \bar{u}$, excrement: Av. giudha-, Phl. güh, NP. guh. anu-, now: Av. nū, Phl. nūn, NP. aknūn. azhnū, knee: Av.

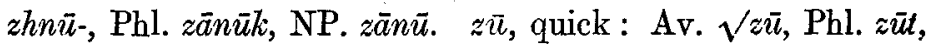
NP. ziùd. būm, earth: Av. būmi-, Phl. būm, NP. būm. $\sqrt{ }$ aparmu $\bar{u}$, hope : Av. a- $\sqrt{ }$ parmū, Phl. -, NP. -

2. $\mathrm{Kj} . \bar{u}=$ Av. $a$, Phl. $a k, \mathrm{NP} . a$.

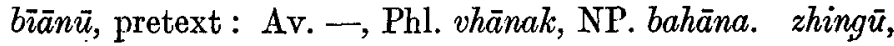
alive: Av. -, Phl. zivvandak, NP. zinda. hamū, all: Av. hama, Phl. hamak, NP. hama. khānū, house (probably loan-word): Phl. khānak, NP. khāna. 
3. Kj. $\vec{u}=$ Av. $a w$, Phl. $a w, a p$, NP. $a b$.

hastür, thick, heavy, strong : Av. stawra-, Phl. stawr, stapr, NP. sitabr.

4. $\mathrm{Kj} \cdot \bar{u}=\mathrm{Av} . a 0, \mathrm{Phl} . \bar{o}, u$, NP. $\bar{o}, \bar{u}$.

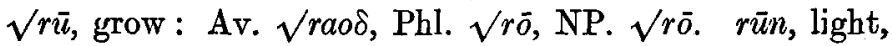
refulgent: Av. raokhshna-, Phl. rōshan, NP. rōshan. rūn, butter: Av. raoghna-, Phl. rōkan, NP. rōghan. $\sqrt{ }$ khurūsh, noise, confusion: Av. khraosh-, Phl. khrust, NP. khurōsh. püch, empty: Av. passh-, Phl. -, NP. püch.

5. $\mathrm{Kj} . \bar{u}=\mathrm{Av} . a v$, Phl. $a v$, NP. $u$.

$\sqrt{ } d \bar{u}$, answer, retort: Av. $\sqrt{ }$ dav, Phl. $\sqrt{ } d a v$, NP. -. $\sqrt{ } c h \bar{u}$, go: Av. $\sqrt{ }$ shav, Phl.,- NP. $\sqrt{ }$ shu.

6. Kj. $\bar{u}=$ Av. $u$, Phl. $u$, NP. $u$.

jūt, a plough : Av. yukhta-, Phl. yukht, NP. juft. sür, red : Av. sukhra-, Phl. sukhr, NP. surkh. süchar, porcupine: Av. sukuruna-, Phl. sukur, NP. sugur.

7. Kj. $\bar{u}=$ Av. $u$, Phl. $\bar{u}, \bar{o}$, NP. $\bar{u}, \bar{o}$.

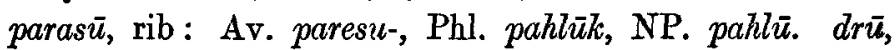
lie: Av. $\sqrt{ } d r u j$, Phl. darōg, NP. durōgh. shī, husband: Av. shudra-, Phl. shō , NP. shī, shō. küderī, where: Av. ku$\theta r a$, Phl. ?, NP. -

\section{XI. $u$ similar to French $u$}

Generally speaking this vowel shows the same equivalents in other languages as does $\bar{u}$, and is sometimes interchangeable therewith.

1. Kj. $\underline{u}=$ Av. $a o$, Phl. $\bar{o}$, NP. $\bar{u}$, aw.

gue, ear : Av. gaosha-, Phl. gōsh, NP. gūsh. suind, oath : Av. saokenta-, Phl. -, NP. sawgand.

2. Kj. $\underline{u}=$ Av. $a$, Phl. $\bar{e}, \mathrm{NP} . \bar{\imath}$. tuzh, sharp : Av. taezha-, Pbl. tēj, NP. tīz.

3. Kj. $\underline{u}=$ Av. va, Phl. $u$, NP. $u$.

khüe, owner : Av. khvadāta-, Phl. khudāi, NP. khudā. 
4. Kj. $\underline{u}=\mathrm{Av} . v a$, Phl. $v \bar{a}, \mathrm{NP} . w \bar{a}$.

$\sqrt{ } k h u i n$, read, sing: Av. $\sqrt{ } k h v a n$, Phl. khvān, NP. $\sqrt{ } k h w \bar{a} n$.

1. $\bar{a} o$.

\section{The Commoner Diphthongs}

In $b \bar{a} o s h$, the upper part of the side, the side of the bosom, $\bar{a} o$ represents Av. aro with loss of $r$, for other examples of which see consonant tables, cf. Av. barozhda-. There does not appear to be any equivalent word in Phl. or NP.

The diphthong $\bar{a} o$ also occurs in $b \underline{l} \bar{a} o$, scattered, dispersed, which has no counterpart as a separate word in either $\mathrm{Phl}$. or NP., of which the origin from Av. para-, OP. parā-, suggests itself. In $k \underline{l} \bar{a} o$, a hat, an equivalent $\bar{a}$ is seen in NP. kulāh, from which it is quite possibly a loan-word.

2. ao.

In the word draosh, shining, glinting, ao represents NP. af in dirafsh. Draosh is probably a NP. loan-word. ao occurs in a number of words of which the etymology is not clear, and in loan-words from Ar., when it usually represents aw,

as in haoz, Ar. حو ض, kihaoti, Ar. خو, etc.

3. ai.

This only occurs in loan-words, as aiwān, NP. aiuān; aib, Ar. 'aib; ain. Ar. 'ain.

4. $i \bar{e}$.

This combination is very common. In loan-words it represents original $\bar{\imath}$, as in giēlass, from NP. gìless, the sweet cherry. In the word kiêrd it represents Av. $a$, Phl., NP. $\bar{a}$ (see also $\bar{e}$ in this value). It occurs in many words as a result of the diminutive $\bar{e} l a$, being added to a word already ending in $i$ or $\bar{\imath}$, as $p s h i \bar{e} \underline{l} a$, a cat $=p s h \bar{\imath}-\bar{e} \underline{l} a, ;$ birsie $\underline{\underline{l}} a$, unripe grapes $=$ bir- $\sqrt{s i-e ̂ l}$ la.

\section{Anaptyxis}

Unlike its neighbour, NP., Kurmanji displays a repugnance to much-vocalized words, and in many cases where NP. has 
introduced anaptyxis, $\mathrm{Kj}$. has perpetuated the original form. This is most noticeable in the case of initial pairs of consonants. Examples : drēzh, Av. drājo-, NP. dirāz. drū, Av. draogha-, NP. durūgh. stēr, Av. star-, NP. sitāra. $\quad \sqrt{ }$ shka, Av. $\sqrt{ }$ schan, NP. $\sqrt{ }$ shik. spī, Av. spaeta-, NP. sifīd. stūn, Av. stūna-, NP. sutūn, and many others. In all these examples Phl. does not show anaptyxis.

$\mathrm{Kj}$. rejects short vowels in initial positions before two consonants, or short vowels between them.

Examples: $\sqrt{ } s p \bar{a} r$, Av. us-V par; slām, Ar.

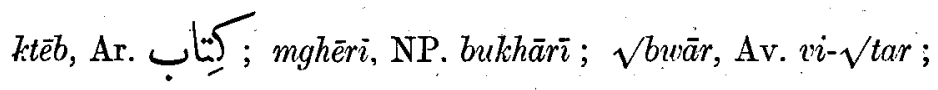
$\sqrt{k r}, A v, \sqrt{ } k a r ; s b e ̀$, Ar. $\tau^{\prime}$, and numerous others.

In many cases $\mathrm{Kj}$. is not averse to a word of two or three consonants entirely unvocalized. Such are $p s r$, a rent or tear ; $p r$, full ; qng, anus; sht, thing; $p c h k$, small ; $f s h k$, a spark; $\sqrt{ } b r z k$, scorch; and many others.

\section{SEMI-VoweI $w$}

1. $\mathrm{Kj} . w=$ Av. $w$, Phl. $f$, NP. $f$. wa, towards, against: Av. aiwi, Phl., NP. af- (in verb forms).

2. $\mathrm{Kj} . w=$ Av. $w, \mathrm{Phl}, w, \mathrm{NP} . b$.

hawr, cloud: Av. awra, Phl. awr, NP. abr.

3. Kj. $w=$ Av. $u, \mathrm{Phl}$. -, NP. - .

chwār, four : Av. chat $w \bar{a} r$.

4. $\mathrm{Kj} . w=$ Av. $v$, Phl. $v, \mathrm{NP} . b$.

ward, a patch of ploughing: Av. $\sqrt{\text { varz, }}$ Phl. varz, NP. barz.

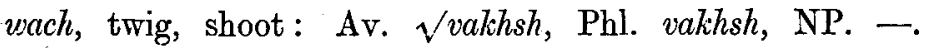
wārān, rain : Av. vāra-, Phl. vāāān, NP. bārān. wafr, snow : Av. vafra, Phl. vafr, NP. barf. wan, tree: Av. vanā-, Phl.

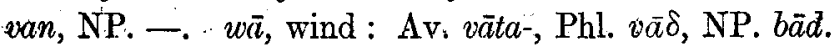


5. $\mathrm{Kj} . w=\mathrm{Av} . v$, Phl. $v$, NP. $v$.

$d \bar{\imath} w$, devil : Av. daava-, Phl. dēv, NP. divv.

6. Kj. $w=$ Av. $\dot{v}$, Phl. $v$, NP. $g$.

wenāsa, violent offence, quarrel : Av. ?, Phl. vinās, NP. gunäh. wer, circular motion: Av. $\sqrt{ }$ vere $\theta$, Phl. $\sqrt{ }$ vart, NP. $\sqrt{ }$ gard. Note also $\mathrm{Kj}$. win, lost, where NP. shows gum; and $\bar{a} w r$, fire, against $\mathrm{Kj}$. ägir.

7. Kj. $w=$ Av. $f$, Phl. $f$, NP. $h$. kew, mountain : Av. kaofa-, Phl. kōf, NP. küh.

8. $\mathrm{Kj} . w=$ Av. $f$, Phl. $f$, NP. $f$.

nāwik, navel : Av. näfah-, Phl. nājak, NP. näf. wer, over, . forward: Av. fra-, Phl. fra-, NP. far-.

$8 a$. Kj. $w=f$ in loan-words.

kawsh, footgear : NP. kafsh. kaugīr, ladle: NP. kafgīr.

9. Kj. $w=$ Av. $p$, Phl. $p, f$, NP. $b, p, v$.

aw, water: Av. āpo-, Phl. $\bar{a} p$, NP. $\bar{a} b . \sqrt{ } n w \bar{\imath}$, write: Av. ni- $\sqrt{ }$ pish, Phl. nipishtan, NP. navishtan. $\sqrt{ }$ rew, go: Av. $\sqrt{ }$ rap, Phl. $\sqrt{ }$ raf, NP. $\sqrt{ }$ raf, $\sqrt{ }$ rav. $-w \bar{a} n$, a guardian : Av. pāna, Phl. -pān, NP. -bān. $\sqrt{ } w a$, flee: Av. $\sqrt{ } p a d$, Phl. -, NP. -

10. Kj. $w=$ Av. $m$, Phl. $m$, NP. $m$.

chaw, eyes: Av. chashman-, Phl., NP. chashm. zāaz, bridegroom: Av. zämātār-, Phl. dämād, NP. dāmād. hāwīn, summer: Av. hāmin, Phl. hāmīn, NP. - nāw, name: Av. nāman-, Phl., NP. nām. n̄̄w, half: Av. nazma, Phl. $n \bar{e} m$, NP. nìm. $\sqrt{ } n w \bar{a}$, display: Ar. ni- $\sqrt{ } m \bar{a}, \mathrm{Phl}, \mathrm{NP}$. $\sqrt{ }$ nimā. nwēzh, prayer: Av. nemah-, Phl. namāch, NP. namāz. This last word is probably a loan-word from $\mathrm{Phl}$. or NP.

10a. Kj. $w=m$ in modern loan-words.

awīn, safe: Ar. مئ hawìr, dough : Ar.

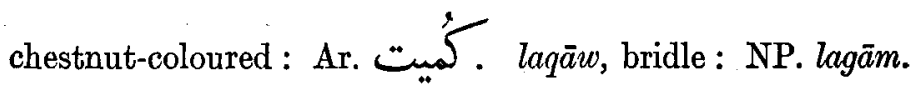


11. Kj. $w=$ Av. $h$, Phl. $k h$, NP. $k h$.

wishk, dry : Av. hushka, Phl., NP. khushk. wash, pleasant : Av. hush, Phl. khvash, NP. khüsh.

Initial $w$ in wirch, a bear, Av. aresha-, may be a change from the $h$ augment of hirch, which is also commonly heard; similar to $h$ to $w$ in wishk (see above), Phl. and NP. both show khirs.

$\sqrt{ } u \bar{\imath} s$, wish, desire: invites comparison with Av. $\sqrt{ } v a s, \sqrt{ } v \bar{z} s$, as apart from Av. hvād, hvāst, and Phl., NP. $\sqrt{ } k h v a \bar{s} t$.

In the word wurd, small, broken small (which also occurs as hur, hurd), $w$ appears to represent $k h$ in Av. $\sqrt{ } k h v a r$, Phl. khurtak, and NP. khurda.

12. $\mathrm{Kj} . w$ as augment.

wushtir, camel : Av. ushtra-, Phl. ushtr, NP. shutur. wulakk, beast of burden: NP. ulägh. wutū, fiat iron: NP. $u t \bar{u}$.

13. Kj. $w=$ Av. $t$, Phl. $t$, NP. $d, \delta$.

$\sqrt{ } b w \bar{a} r$, crossing over: Av. vi- $\sqrt{ }$ tar, Phl. $\sqrt{ }$ vitar, NP.

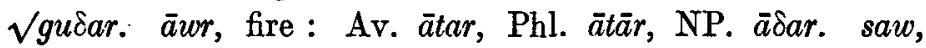
hundred : Av. sata-, Phl. sat, NP. sad.

The words $b w \bar{a} r$ and $\bar{a} u r$ are seen also as $b g \bar{a} r$ and $\bar{a} g i r$, and may be developments therefrom. See XVI, 6 .

\section{XIII. $y$}

This letter is rare, and is usually seen only in loan-words, as yākhud, NP. y $\bar{a} k h u d ; y a s \bar{a} k h$, Tk. yas $\bar{a} k h$. It appears as an augment in yakhsir, a prisoner of war : Ar. اسير, but this is probably borrowed from Oriental Tk. yesīr.

\section{CONSONANTS}

$\begin{array}{llllll}\text { Guttural } & k & k h & g & g h & q \\ \text { Palatal } & c h & j & & & \\ \text { Dental } & t & d & & & \\ \text { Labial } & p & f & b & m \\ \text { Nasal } & n & \bar{n} & n g & \end{array}$




$\begin{array}{lllll}\text { Liquids } & r & \underline{r} & l & \underline{l} \\ \text { Sibilant } & s & s h & z & z h \\ \text { Aspirate } & h & \underline{h} & & \end{array}$

\section{Gutturals}

XIV. $k$

1. Kj. $k=$ Av. $k$, Phl. $k$, NP. $k$.

kamar, rock: Av. kamarä-, Phl., NP. kamar. $\sqrt{ }$ kan, dig, carve: Av. $\sqrt{k a n, ~ P h l ., ~ N P . ~} \sqrt{ }$ kan. wishk, dry: Av. hushka, Phl., NP. khushk. tak, rutting: Av, taka-, Phl., NP. $t a k$.

2. Kj. $k=$ Av. ?, Phl. kh, NP. $k h$.

kirīn, to buy: Av. ?, Phl. kharītan, NP. kharìdan.

3. $\mathrm{Kj} . k=\mathrm{Av} . h, \mathrm{Phl}$. kh, NP. kh.

kèm, pus, temper : Av. haəm, Phl. khēm, NP. khīm.

4. $\mathrm{Kj} . k=\mathrm{Av} . k h$, Phl. $k h, \mathrm{NP} . k h$.

kāni, spring, source: Av. khan-, Phl. khānik, NP. khänī. kar, ass: Av. khara-, Phl., NP. khar. $\sqrt{ }$ kand, laugh : Av. $\sqrt{ }$ khvand, Phl., NP. $\sqrt{ }$ khand.

5. Kj. $k==$ Av. $c h$, Phl. $k$, NP. $k$.

kerā, spawn: Av. chïra, Phl., NP. -. $\sqrt{ }$ shka, break : Av. $\sqrt{ }$ scha, Phl. $\sqrt{ }$ shika, NP. $\sqrt{ }$ shika.

6. Kj. $k=$ Av. $g$, Phl. $g$, N.P. $g$.

kirch, wrinkle : Av. $\sqrt{ }$ garcz, Phl., NP. -- kisht-, finger : Av. angushta-, Phl. angust, NP. angusht.

$\mathrm{XV} . \quad k h$

1. $\mathrm{Kj} . k h=\mathrm{Av} . h v, \mathrm{Phl} . k h u$, NP. $k h u$.

kho, self: Av. hva-, Phl., NP. khud (Av. hvatō). khēz, relatives: Av. hvaotush-, Phl., NP. khvēsh. khue, owner:

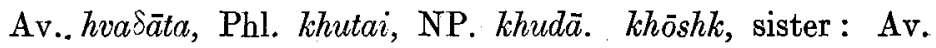
hvanhar, Phl., NP. khvāhar. khur, sun: Av. hvare-, Phl., NP. khür.

The influence of the $v$ in $h v$ is seen in most of the vowels following the $\mathrm{Kj}$. kh. 
2. Kj. $k h=$ Av. $k$, Phl. $k$, NP. $g$. sikhur, a porcupine: Av. sukuruna-, Phl. sukur, NP. sugur.

3. $\mathrm{Kj} . k h==g h$ in loan-words.

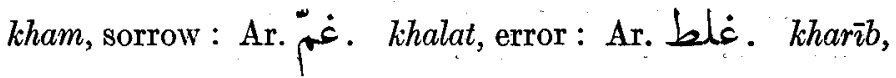

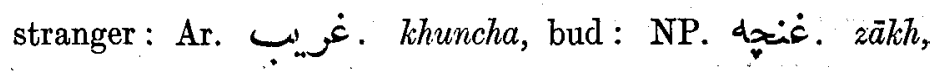
alum : Ar. NP. غاغ j. bākhcha, garden: NP. باغِ.

4. $\mathrm{Kj} \cdot k h=\mathrm{Ar} \cdot \tau$.

khaml, body ornaments: Ar. Jo khenã, henna: Ar. 5. $. \mathrm{Kj} . k h=\mathrm{Tk}$. and North Kj. $q$, Ar. $q$.

khanjilla, a pretty, small child: Tk., North Kj. qenj.

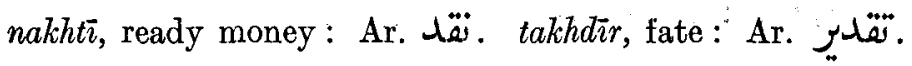

\section{XVI. $g$}

1. Kj. $g$ (initial) $=$ Av. $g$, Phl. $g$, NP. $g$.

ga, time, place: Av. gātu-; Phl. gãs, NP. gāh. gan, fetid : Av. gainti-, Phl. gand, NP. gand. ganum, wheat: Av. gantaoma-, Phl. gantum, NP. gandum. germ, warm: Av. garema-, Phl., NP. garm. ger, hill : Av. gairi, Phl. —, NP.—. $g \bar{a}$, ox : Av. $g \bar{a} v-$, Phl. gav, NP. gā $v$. gāw, pace: Av. gāma, $\mathrm{Phl}$, NP. gām. girīfān, pouch, pocket: Av. garēua-, Phl. garīopān, NP. girībān.

2. $\mathrm{Kj} . g=\mathrm{Av} . k$, Phl. $g$, NP. $g$. -ger, a worker in, maker of : Av. -kara, Phl., NP. gar.

3. Kj. $g=$ Av. $k$, Phl. ?, NP. $k$.

gōza, earthen pot: Av. kauza-, NP. kūza.

4. $\mathrm{Kj} \cdot g=$ Av. $v$, Phl. $g$, NP. $g$.

gurch, kidney: Av. veretka-, Phl. gurt, NP. gurda. $\sqrt{ }$ gür, change: Av. $\sqrt{ }$ varet, Phl. gāsh, NP. $\sqrt{ } g \bar{a} s h, y \bar{a} r$. gurg, 
wolf: Av. vehrka-, Phl., NP. gurg. gund, village: (Skt. venda-), Phl. gund, NP. ghund.

5. Kj. $g=$ Av. v, Phl. $v$, NP. $g$.

$\sqrt{ }$ gar, turn, wander: Av. $\sqrt{ }$ varet, Phl. $\sqrt{ }$ vart, NP. $\sqrt{ }$ gard. gish-k, all: Av. vispo-, Phl. visp, NP. -.

6. $\mathrm{Kj} . g=$ Av. $t$, Phl. $t, \mathrm{NP} . d, \delta$.

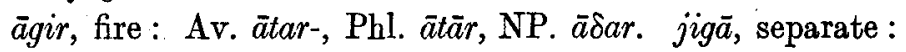
Av. yuta-, Phl. jutāk, NP. judā. reg, line, row : Av. areta-, Phl. -, NP. rada.

Compare also zerg, yellow, with NP. zard; tagbir, loanword from Ar. بـ ; pīāga, loan-word from NP. pīāda.

7. Kj. $g=$ Av. $g h$, Phl. ?, NP. $g h$. mërg, turf, meadow : Av. maregha-, Phl. ?, NP. margh.

8. Kj. $g i=\mathrm{Av} \cdot g a$, Phl. $j, \mathrm{NP} . j$.

giān, soul : Av. gaza-, Phl., NP.jān.

XVII. $g h$

The sound is rarely heard except in loan-words, usually having been hardened to $k h$ (which see). It appears in the place-name Mughägh, a low-lying marshy place, which is a loan-word from NP. mughāk.

In the loan-word mghēri, it represents NP. $k h$ in bukhāri

\section{XVIII. $q$ (Ar. ق)}

1. Kj. $q=$ Av. $k$, Phl. $k$, NP. $k$.

qurna, corner, edge : Av. karana-, Phl. kanārak, NP. kanār. $q \bar{\imath} n$, anger : Av. kaana-, Phl. kēen, NP. kīn. quz, vulva : Av.?, Phl. ?, NP. kus. qng, anus : Av. ?, Phl., NP. kün.

$k$ is seen unchanged in kun, a hole. There are several cases where, although $k$ is seen changed to $q$, the original form is still in use, as in pishqil, pishkil; qaladar, kaladar; $q \bar{o} \underline{l}, k \bar{o} \underline{l} ;$; $q \bar{a} l i q, k \bar{a} l i k$.

2. Kj. $q=$ Av. $g$, Phl. $g$, NP. $g$.

qurg, gullet : Av. garah-, Phl.garük, NP. gulü. qizh, locks : 
Av. gaēsa-, Phl. gès, NP. gès. qirch, wrinkle: Av. V Varez-, Phl., NP. - .

The last word is also seen as girj, chirch, and kirch.

3. Kj. $q=k h$ in loan-words.

qiqua, dotard : Ar. خرف qirzhānk, crab : NP. kharchang.

Palatal.

XIX. ch

1. $\mathrm{Kj} . c h=\mathrm{Av} . c h, \mathrm{Phl} . c h, \mathrm{NP} . c h$.

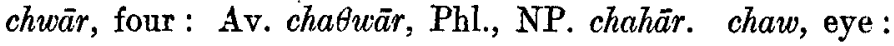
Av. chashman, Phl., NP. chashm. $\sqrt{ }$ chè, cultivate, sow: Av. $\sqrt{ }$ chay, Phl. $\sqrt{ }$ chī, NP. $\sqrt{ } c h \bar{\imath}$. chār, face: Av. chiera-, Phl. chihrak, NP. chihra.

2. Kj. $c h=$ Av. sh, Phl. $s$, NP. $s$. hirch, airch, bear: Av. aresha-, Phl., NP. khirs.

3. Kj. $c h=$ Av. $s h$, Phl. $s h$, NP. $s h$. gōchka, ear: Av. gaosha-, Phl., NP. gōsh.

4. Kj. $c h=$ Av. $s$, Phl. $s$, NP. $s$.

$\sqrt{ }$ chri, call, cry : Av. $\sqrt{ }$ sru, Phl. $\sqrt{ }$ sru, NP. $\sqrt{ }$ surū. pach-, cattle : Av. pasu-, Phl. -, NP.-.

5. Kj. $c h=\mathrm{Av} . s, \mathrm{Phl} . z, \mathrm{NP} . s$. pirch, locks : Av. varesa-, Phl. varz, NP. gares.

6. Kj. $c h=$ Av. --, Phl. sh, NP. sh.

chaqāl, jackal : (Skt. srgāla-), Phl., NP. shaghāl.

7. Kj. $c h=$ Av. z, $\delta$, Phl. - , NP. -

chirch, wrinkles: Av. $\sqrt{ }$ gare $\delta$, garez. china, chin: Av. zanva- (cf. NP. zanakh).

8. Kj. $c h=$ Av. $k$, Phl. $k$, NP. $g$.

suchar, porcupine: Ar. sukuruna-, Phl. sukur, NP. sugur. לkerā, shears: Av. $\sqrt{ }$ leret, Phl., NP. -

9. $\mathrm{Kj} . c h=$ Av. $g$, Phl. -, NP. - .

chirch, wrinkles : Av. $\sqrt{ }$ garez, Phl., NP. -

JRAS. APRIL 1922. 
10. $\mathrm{Kj} . c h=$ Av. $t$, Phl. $t, \mathrm{NP} . d$.

gurch, kidney: Av. veretka-, Phl. gurtak, NP. gurda.

11. Kj. $c h=\mathrm{Av}$. ?, Phl. ?, NP. kh.

chilm, mucus : Av. ?, Phl.?, NP. khilm.

12. Kj. $c h=A \nabla$. ?, Phl. ?, NP. $k$.

kēch, flea: Av. ?, Phl. ?, NP. kēk.

\section{XX. $j$}

1. $\mathrm{Kj} \cdot j=$ Av. $j$, Phl. $j, z$, NP. $z$.

jind $\bar{u}$, alive: Av. $\sqrt{ } j i$, Phl. zivvandak, NP. zinda. ārejū, desire: Av. $\sqrt{ } \operatorname{ar} \bar{e} j$, Phl. arj, NP. $\bar{a} r z \bar{u} .-j \bar{a} r$, a place of : Av. ?, Phl. jār, NP. $z \bar{a} r$.

2. Kj. $j=$ Av. $y$, Phl. $y, j$, NP. $j$.

jüt, plough: Av. yukhta-, Phl. yukht, NP. juft. jēzhn, festival: Av. yasna-, Phl. yashn, NP. jashn. jerg, liver: Av. yākar-, Phl. jakar, NP. jigar. jig $\bar{a}$, separate: Av. yuta-, Phl. jutāk, NP. judā. jūīn, to chew: Av. ?, Phl. jütan, NP. jāvīdan.

3. $\mathrm{Kj} \cdot j=\mathrm{Av} . c h$, Phl. ch, NP. $z$. rōj, light, day: Av. raochah-, Phl. roch, NP. rūz.

4. Kj. $j=$ Av. $g$, Phl. $g$, NP. $g$. $j \bar{a}$, time : Av. gātu-, Phl. gās, NP. gāh.

5. Kj. $j=$ Av. ? (Skt. $t$ ), Phl. -, NP. $d$. janjar, a threshing appliance: Skt. yantrá-, Phl. NP. jandara.

\section{Dental \\ XXI. $t$}

1.Kj. $t=$ Av. $t$, Phl. $t$, NP. $t$.

tüw, seeds: Av. taokhma-, Phl. tokhm, NP. tukhm. $\sqrt{ } t w$, melt, flow: Av. $\sqrt{ }$ tach, Phl. $\sqrt{ }$ vitākh (NP. gudākhtan). stēr, star : Av. star-, Phl. stārak, NP. sitāra. tur, hatchet: Av.?, Phl. tabrak, NP. tabar. jüt, plough: Av. yukhta-, Phl. yulht, NP. juft. $\sqrt{ } t \bar{a} s h$, carve, cut: Av. $\sqrt{ }$ tash, Phl. $\sqrt{ }$ tash, NP. $\sqrt{ }$ taräsh. 
2. Kj. $t=$ Av. $t$, Phl. $t$, NP. $d$.

nwt, felt: Av. nimata-, Phl. namat, NP. namad.

3. Kj. $t=$ Av. $d$, Phl. $d$, NP. $d$.

tush, difficulty, obstruction: Av. duzh-, dush-, Phl. dush-, NP. dush-.

4. Kj. $t=d$ in loan-word.

nakhte, some: Ar. نقe.

5. $\mathrm{Kj} . t=\mathrm{Av} . c h, \mathrm{Phl} . c h, \mathrm{NP} . z$.

$\sqrt{ }$ süt, burn : Av. $\sqrt{ }$ such, Phl. sōc, NP. $\sqrt{ }$ sōz.

6. Kj. $t=$ Av. $s$, Phl. ?, NP. ch.

püt, empty, rotten, rubbish : Av. paosh-, Phl. ?, NP. püch.

Nos. 5 and 6 are more fully exemplified in Northern Kj.

XXII. $d$

1. $\mathrm{Kj} . d=\mathrm{Av} . d, \mathrm{Phl} . d, \mathrm{NP} . d$.

dān, tooth : Av. dantan, Phl. dantān, NP. dandān. danik,

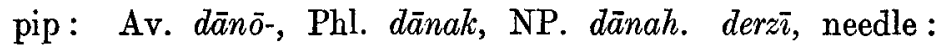
Av. $\sqrt{ }$ darez, Phl. darz, NP. darzī (tailor). $\sqrt{ }$ der, tear : Av. $\sqrt{ }$ dar, Phl., NP. $\sqrt{ }$ dar. $\sqrt{ }$ da, give: Av. $\sqrt{ } d \bar{a}$, Phl., NP. $\sqrt{ } d a$.

2. Kj. $d=$ Av. j, Phl. $z$, NP. $z$.

$\sqrt{ } d a$, strike: Av. $\sqrt{ }$ jan, Phl., NP. $\sqrt{ }$ zan. Compare also Kj. $\sqrt{ } d o z$, seek, find, with NP. justan from OP. $\sqrt{ } j a d$.

3. In the following two words-dast, hand, dost, friend-lie one of the incongruities of $\mathrm{Kj}$. As original $z$ does not become $d$ it would appear that dast and dost must $(a)$ have been borrowed from $\mathrm{Phl}$. or $\mathrm{NP}$. if $\mathrm{Kj}$. is a non-Persian language, and supplanted the words zast and zost, which would have followed Av. zasta- and zaosha-, (b) be original words in a Persian language.

A further difficulty is, however, presented by the word ward, a cultivated plot, against which $z$ is seen in Av. $\sqrt{ }$ varz, Phl. $\sqrt{ }$ varz, NP. $\sqrt{ }$ barz. 


\section{- Labiatus \\ XXIII. $p$}

1. $\mathrm{Kj} \cdot p=\mathrm{Av} \cdot p$, Phl. $p$, NP. $p$.

$\sqrt{ }$ aparmū, trust, hope: Av. $\bar{a}-\sqrt{ }$ parmū, Phl., NP. spī, white: Av. spaēta, Phl. spēt, NP. safīd. spār, trust, entrust: Av. us- $\sqrt{ }$ par, Phl. $\sqrt{ }$ spar, NP. $\sqrt{ }$ sipār. pas, small cattle: Av. pasu-, Phl. pah, NP. - parasū, ribs: Av. paresu-, Phl. pahlūk, NP. pahlū. pert, bridge: Av. parotu-, Phl. puhl, NP. pul. pursha, sleet: Av. parshva-, Phl. parashveh, NP. -

2. $\mathrm{Kj} \cdot p=\mathrm{Av} \cdot p$, Phl. $p$, NP. $b$.

pai, to: Av. paiti, Phl. pat, NP. ba.

3. $\mathrm{K} \mathrm{j} \cdot p=b$ in loan-words.

pirinj, rice: NP. birinj. sharäp, wine: Ar. word borrowed through NP. sharāb. kharāa, bad : Ar. خراب. tapl, drum : Ar. طبل

4. Kj. $p=$ Av. $v$, Phl. $v$, NP. $g$ (see also Kj. b, 3 and 4). pirch, locks: Av. varesa-, Phl. varz, NP. gars. $\sqrt{ }$ psr, break, apart: Av. vi- $\sqrt{ }$ sard, Phl. visastan, NP. gusistan. $\sqrt{ }$ pshè w, being confused, perturbed : ? Av. vi- $\sqrt{ } k h s h \bar{\imath} w, \mathrm{Phl}$., NP. -.

5. $\mathrm{Kj} \cdot p=f$ in loan-words.

sipla, humble, mean: Ar. dle. pata, the Ar. proper name $C^{\ddot{\theta}}$

\section{XXIV. $b$}

( $b$ is seldom seen except initially, changing to $w$ medially and finally.)

1. Kj. $b=$ Av. $b$, Phl. $b$, NP. $b$.

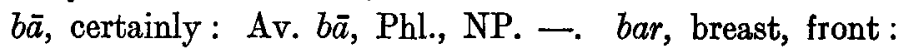
Av. para-, Phl., NP. - berz, high : Av. baraz, Phl. burz, NP. -. brā, brother : Av. brätar, Phl. brätar, NP. berādar. 
bin, the bottom: Av. buna-, Phl., NP. bun. bizin, goat: Av. buja-, Phl. buj, NP. buz.

2. $\mathrm{Kj} . b=$ Av. $v$, Phl. $v$, NP. $b$.

bafr, snow: Av. vafra-, Phl. vafr, NP. barf. bēn, snout :

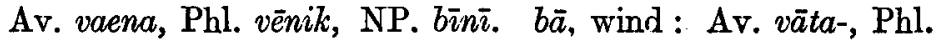
$v \bar{a} t$, NP. $b \bar{a} d$.

3. $\mathrm{Kj} . b(i)=$ Av. $v(i)$, Phl. $v(i)$, NP. $g u$.

$\sqrt{b u t a ̄ r, ~ p a s s i n g ~ o v e r: ~ A v . ~ v i-~} \sqrt{ }$ tar, Phl. $\sqrt{ }$ vitar, NP. gudhar. $\sqrt{ } b z h a \bar{r}$, choosing from : Av. vi- $\sqrt{ }$ char, Phl. $\sqrt{ } v c h a r$, NP. $\sqrt{ }$ guzar. bināsa, quarrel, violent offence : (Skt. vi- $\sqrt{ }$ naç), Phl. vinās, NP. gunāh. barāz, hog: Av. varāza-, Phl. varāz, NP. gurāz.

4. Kj. $b=$ Av. ?, Phl. $g$, NP. $g$.

hirs, hunger: Av. ?, Phl. gursak, NP. gurs.

5. $\mathrm{Kj} . b=\mathrm{Av} . f, \mathrm{Phl} . f, \mathrm{NP} . f$.

bar, cause, reason: Av. fra-, Phl. freh, NP. firih. bar, loose, free: Av. fra-, Phl. fräj, NP. firāz.

\section{XXV. $f$}

1. $\mathrm{Kj} \cdot f=$ Av. $f$, Phl. $f$, NP. $f$.

ferā, broad, ample: A $\sqrt{ }$ früsh, sell : Av. fra- $\sqrt{ }$ vakhsh, Phl. ferüsh, NP. ferüsh.

2. Kj. $f=$ Av. $f$, Phl. $f$, NP. $h$.

kêf, mountain : Av. kaufa-, Phl. kōf, NP. kūhh.

3. $\mathrm{Kj} . f=$ Av. $p$, Phl. $p$, NP. $f$.

$\sqrt{ }$ kaft, fall : Av. $\sqrt{ }$ pat, Phl. $\sqrt{ } k a f t$, NP. $\sqrt{ } \bar{o} t$.

4. $\mathrm{Kj} . f=$ Av. $p$, Phl. $p$, NP. $p$.

$\sqrt{ }$ fer, fly: Av, parena-, Phl., NP." $\sqrt{ }$ par.

5. $\mathrm{Kj} \cdot f=\mathrm{NP}$. $k h$.

fēnūk, cool : NP. khun ük.

XXVI. $m$

1. Kj. $m=$ Av. $m$, Phl. $m$, NP. $m$.

mēsh, fly: Av. makhshi-, Phl. magas, NP. magas. pim, fat: Av. paeman, Phl. pim (NP. pī). nim, dampnęss : 
Av. namna-, Phl., NP. nam. maz, mas, large: Av. mas, Phl. mas, NP. mih. māsī, fish : Av. masya-, Phl. māhik, NP. $m \bar{a} h \bar{\imath}$.

2. Kj. $m=$ Av. $\bar{u}$, Phl. $v$, NP. $b$. $z m \bar{a} n$, tongue: Av. hizū-, Phl. uzvān, NP. zabān.

3. $\mathrm{Kj} . m=b$ in loan-words.

ghumār, mist: Ar. غبا. mghērin, stove: NP. mhāna, pretext : NP. dil.?.

4. Kj. $m=$ Av. $n g$, Phl. $n g$, NP. $n g$. amust, finger : Av. angushta, Phl. angust, NP. angusht.

\section{NASAL \\ XXVII. $n$}

1. Kj. $n=$ Av. $n$, PhI. $n$, NP. $n$.

ganum, wheat: Av. gantaoma-, Phl. gantum, NP. gandum. chan, some : Av. chvant-, Phl. chvant, NP. chand. nawa, offspring: Av. napa, Phl. näf, NP. navädah: nēr, male : Av. nairya-, Phl., NP. nar. nizm, low: Av. nitem, Phl., NP. - . $\sqrt{ } n w \bar{a}$, display: Av. ni- $\sqrt{ } m \bar{a}$, Phl. $\sqrt{ } n i m \bar{a}, \mathrm{NP}$. $\sqrt{ }$ imä.

2. Kj. $n=$ Av. $m$, Phl. $n$, NP. $n$.

han-, verbal prefix: Av. ham, Phl., NP. an-. shin, lamentation: Av. khshim-, Phl. shin, NP. -

3. Kj. $n=$ Av. ?, Phl. $n$, NP. $m$.

bān, roof : Av. ?, Phl. bāan, NP. bām.

\section{XXVIII, $\bar{n}$}

I have used this symbol to indicate nasalization following a long vowel. The sound is nearly the same as that heard in Urdu, but is less marked. There are but few words in Kj. which possess it.

The NP. words tāz $\bar{\imath}$ greyhound, $t \bar{a} j$ crown, $b \bar{a} z \bar{\imath}$ gaming, have been borrowed and nasalized to $t \bar{a} \bar{n} z h \bar{\imath}, t \bar{a} \bar{n} j$, and $b \bar{a} \bar{n} z \bar{\imath}$. 
Other words in which it occurs are kōnder, where, for kūder ; lawēñderī, there, for lawēderī ; lā̄ngir, a partisan, for lāgir ; spīñdār, poplar, for spīdār ; and possibly Kurmān̄j, for $\operatorname{Kur}(d)-m \bar{a} d$.

\section{XXIX. $n g$}

This is the guttural nasal, pronounced as in English " hang", " bang".

1. Kj. $n g=$ Av. $n$, Phl. -, NP. - .

māng, moon: Av. maonha-, māh, Phl., NP. māh.

2. Kj. $n g=$ A . $n-g$, Phl. $n-g$, NP. $n-g$.

angust, finger: Av. angushta-, Phl. angust, NP. angusht. angwēn, honey: Av. ?, Phl. angumēn, NP. angubin. rang, colour: (Skt. ranga-), Phl. -, NP. rang.

3. Kj. $n g=$ Av. ?, Phl. $n$, NP. $n$.

$q n g$, anus: Av. ?, Phl., NP. kūn. Loan-word kānga, a mine: NP. kān. Loan-word dānga, a pimple: NP. dāna. 4. Kj. $n g=\mathrm{Av} . n t, \mathrm{Phl} . n t, \mathrm{NP} . n d$. chang, some: Av. chvant, Phl. chvant, NP. chand. pang, idea : Av. pantan, Phl., NP. pand.

5. Kj. $n g==$ Av. $n d$, Phl. $n d$, NP. $n d$.

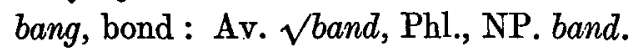

6. $\mathrm{Kj} . n g=n d$ in loan-word.

$$
\begin{gathered}
\text { mangīl, kerchief : Ar. Jis. } \\
\text { LIQUIDS } \\
\text { XXX. } r
\end{gathered}
$$

1. Kj. $r=$ Av. $r$, Phl. $r$, NP. $r$.

asr, tears: Av. asru, Phl. ars, NP. ars. ägir, fire: Av. ätar-, Phl. àtār, NP. ädhar. bar, a reason : Av. fra-, Phl. freh, NP. firih. hushtir, camel: Av. ushtra, Phl. ushtir, NP. shutur. $\sqrt{ }$ ressh, flow: Av. $\sqrt{ }$ rech, Phl., NP. $\sqrt{ } r \bar{z} z$. rān, thigh: Av. rāna-, Phl., NP. rān.

2. Kj. $r=$ Av. $r$, Phl. $r$, NP. $l$.

gerū, gullet: Av. garah-, Phl. garūk, NP. gulū. 


\section{XXXI. $\underline{r}$ (alveolar, strongly trilled)}

The use of $\underline{r}$, though quite distinct from that of $r$, does not appear to be the subject of any readily detected rule. In the words par feather, $\sqrt{ }$ bir cut, the $\underline{r}$ has its counterpart in NP. In other words, such as $p r$, full, many : Av. per na, Phl., NP. pur; rawa, a drove: NP. rama; $\sqrt{ }$ rif, snatch away: NP. $\sqrt{ } r u b ;$ rash, black: NP. rakhsh (Firdausi), the cause of its strengthening is not evident. The sound is very widely used, and is one of the commonest characteristics of the language.

\section{XXXII. $l$}

1. $\mathrm{Kj} . l=$ Av. $r$, Phl. $l$, NP. $l$.

giti, complaint: Av. gereza-, Phl. gilak, NP. gileh.

2. Kj. $l=$ Av. $r$, Phl. $r$, NP. $r$.

gil, detention, holding: Av. $\sqrt{ }$ garw, Phl. $\sqrt{ }$ graf, NP. gir. halata, hill : Av. haraiti-, Phl. har, NP. -

3. Kj. $l=$ Av. $n$, Phl. $n$, NP. $n$.

kul, blunt: Av. ? (Skt. kuntha-), Phl. ?, NP. kund. jūāl, young, pretty: Av. yuvan, Phl. javānak, NP. javān. māl, house: Av. nmana-, Phl. mān, NP. mān. ruwāla, rōla, beloved, soul : Av. urvan-, Phl. ruvān, NP. rawān. zil, large, powerful, heavy: Av. zainti-, Phl. zand, NP. - la, at upon, from : Av. ana, Phl., NP. -

4. Kj. $l=$ Av. $t$, Phl. $t$, NP. $t$.

kul, short: Av. kutāha-, Phl. kūtāk, NP. kütāh.

5. Kj. $l=$ Av. $t$, Phl. $d$, NP. $d$.

khulā, God : Av. khvadhäta-, Phl. khudāk, NP. khudā.

6. $\mathrm{Kj} . l=d$ in loan-word.

klìl, key: NP. kilīd.

\section{XXXIII. $\underline{l}$}

Similar to the Russian $l$

1. Kj. $l=$ Av. $r$, Phl. $r$, NP. $r$.

gulch, kidney: Av. veretka-, Phl. gurtak, NP. gurda. dō̄ vale: Av. darenā, Phl. ?, NP. darah. $\sqrt{ } k \bar{e} \underline{l}$, cultivation : 
Av. $\sqrt{ }$ karsh, Phl. $\sqrt{ } k \bar{a} s h, k a ̈ r$, NP. $\sqrt{ } k a \bar{r}$. sipul, spleen : Av. spereza-, Phl. sparz, NP. sipurz.

2. $\mathrm{Kj} . \underline{l}=\mathrm{Av} . r$, Phl. $l$, NP. $l$.

sāl. year : Av. sareta-, Phl., NP. sāl. $\sqrt{ } m a \bar{l}$, rub, sweep : Av. $\sqrt{ }$ marz, Phl., NP. $\sqrt{ } m \bar{a} l . \quad \sqrt{ } h \bar{e} \underline{l}$, allow: Av. $\sqrt{ }$ harz, Phl., NP. $\sqrt{ } h i \bar{l}$.

\section{SibILANT \\ XXXIV. $s$}

1. Kj. $s=$ Av. $s$, Phl. $s$, NP. $s$.

asr, tears: Av. asru-, Phl.; NP. ars. sipul, spleen : Av. spereza-, Phl. sparz, NP. sipurz. sāl, year: Av. sareta-, Phl., NP. säl. est, bone: Av. asta-, Phl. ast, NP. ust-. hastūr, thick, heavy : Av. stawra-, Phl. stapr, NP. sitabr.

2. Kj. $s=\mathrm{Av} . s$, Phl. $s h, \mathrm{NP} . s h$. ispi, louse: Av. spish-, Phl. shupush, NP. shipish. kës-, tortoise: Av. kasyapa-, Phl. ?, NP. kashaf.

3. Kj. $s=$ Av. $s h$, Phl. $s, s h$, NP. $s h$.

añust, finger: Av. angushta-, Phl. angust, NP. angusht. mist, fist: Av. mushti-, Phl., NP. musht.

4. Kj. $s=$ Av. $\theta(r)$, Phl. $s$, NP. $s$.

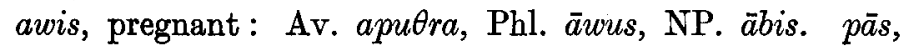

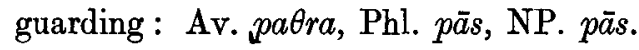

5. Kj. $s=$ Av. $z$, Phl. $z$, NP. $z$.

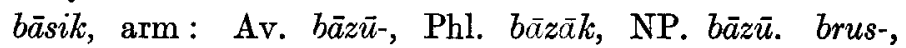
flashing, sparks: Av. $\sqrt{ }$ braz, Phl., NP. -

6. $\mathrm{Kj} . s=z$ in loan-word.

temīs, clean: Ar. تميز.

7. Kj. $s=$ Av. $s$, Phl. $h$, NP. $h$.

pas, sheep, small cattle: Av. pasu-, Phl. pah, NP. $\bar{a} s i k$, gazelle: Av. $\bar{a} s u-, \mathrm{Phl} . \bar{a} h i k, \mathrm{NP}$. $\bar{a} h \bar{u} . \quad m \bar{a} s \bar{\imath}$, fish : Av. masya-, Phl. māhik, NP. māhī.

8. $\mathrm{Kj} . s=\mathrm{Av} . s(z)$, Phl. $s, \mathrm{NP} . h$.

mas, great: Av. mas-, maz, Phl. mas, NP. mih. āsin, 
iron : Av. -, Phl. āsīn, NP. ähan. benāsa, quarrel, offence of violence: Av. -, OP. vi-nätha, Skt. vi- $\sqrt{ }$ nac, Phl. vinās, NP. gunāh.

\section{XXXV. sh}

1. $\mathrm{Kj} . s h=\mathrm{Av}$. sh, Phl. sh, NP. sh.

drawsh, awl : Av. drafsha-, Phl. drafsh, NP. dirafsh. dush, last night: Av. daoshatara-, Phl. dosh, NP. dosh. hishk, dry: Av. hushka-, Phl., NP. khushk. hushtir, camel : Av. ushtra-, Phl. ushtir, NP. shutur. nishīw, precipice: Av. nikhshvaepa-, Phl. nishēp, NP. nishīb. pursha, sleet: Av. parshva-, Phl. parashveh, NP. -. $\sqrt{ }$ sht, wash : Av. $\sqrt{ }$ shud, Phl., NP. $\sqrt{ }$ shust. shä, glad, rejoicing: Av. $\sqrt{ }$ shā, Phl. shät, NP. shād.

2. $\mathrm{Kj} . s h=$ Av. $s$, Phl. $s$, NP. $s$.

$\bar{a} s h$, millstone: Av. as-man, Phl. as-yāw, NP. $\bar{a} s-y \bar{a} b$. gisht, all: Av. vispo-, Phl. -vīst, -vīsp, NP. -. èsh, pain, hurt: Av. yaska-, Phl. yask, NP. jask.

3. Kj. $s h=$ Av. $s$, Phl. $s$, NP. $s h$.

lash, dead flesh : Av. nasu-, Phl. nasāy, NP. lāsh.

4. Kj. $s h=s$ in loan-words.

yakhshīr, prisoner of war : Ar. loan-word through Tk. سبر.

5. $\mathrm{Kj}$. $s h=\mathrm{Av}$. ch, Phl. ch, NP. 一, z.

-sh, enclitic meaning, and, also: Av. -cha, Phl. -ch-, NP.-. $\sqrt{ }$ rish, outpour: Av. $\sqrt{ }$ rech, Phl. $\sqrt{ }$ rēch, NP. $\sqrt{ }$ rīz.

6. $\mathrm{Kj} . \mathrm{sh}=\mathrm{Av} .-$, Phl. $h$, NP. $h$.

$k \bar{a} s h$ (in kāshkesshān), chaff : Av. ?, Skt. kāaca, Phl., NP. kīa .

7. $\mathrm{Kj} . s h=\mathrm{Av} . z$, Phl. $z$, NP. $z$.

messhik, marrow, brain : Av. mazga-, Phl. mazg, NP. maghz.

8. $\mathrm{Kj} . s h=\mathrm{Av} . z, \mathrm{Phl} . s h$, NP. $s h$. $\sqrt{ }$ dossh, milk: Av. $\sqrt{ } d u z$, Phl., NP, $\sqrt{ } d \bar{s} s h$.

9. Kj. $s h=$ Av. sch, Phl. $s$, NP. $s$.

pāsh, after, behind : Av. pascha, Phl. pas, NP. pas. shīen, traces, place, consequences : Av.? $\sqrt{ } s c h a, \mathrm{Phl} ., \mathrm{NP} .-$ 


\section{XXXVI. $z$}

1. Kj. $z=$ Av. $z$, Phl. $z$, NP. $z$.

$\sqrt{ } z a$, be born: Av. $\sqrt{ } z a n$, Phl., NP. $\sqrt{ } z \bar{a}$. barāz, boar: Av. varāza-, Phl. varāz, NP. gurāz. mīz, urine: Av. $\sqrt{ }$ maez, Phl. $\sqrt{ } m \bar{e} z$, NP. $\sqrt{ } m \bar{z}$. zamān, tongue: Av. hizū-, Phl. $u z v \bar{a} n$, NP. $z a b \bar{a} n$.

2. Kj. $z=$ Av. $z$, Phl. $d$, NP. $d$.

$\sqrt{ } z \bar{a} n$, know: Av. $\sqrt{ } z \bar{a} n$, OP. $\sqrt{ } d \bar{a} n$, Phl., NP. $\sqrt{ } d \bar{a} n$. $z \bar{a} u \bar{a}$, bridegroom: Av. zāmātar, Phl. damādh, NP. dāmāad. $a z$, I : Av. azem, OP. adam, Phl., NP. - zer, heart: Av. zered-, Phl., NP. dil.

3. Kj. $z=$ Av. $z h$, Phl. $z, z h$, NP. $z$.

diz, thief: Av. daozhda-, Phl. duzhd, NP. duzd. muz, wage: Av. mizhda-, Phl., NP. muzd.

4. Kj. $z=$ Av. j, Phl. j, NP. $z$.

bizin, goat: Av. buja-, Phl. buja, NP. buz. $\sqrt{ } z a r$, bray : Av. $\sqrt{j}$ ar, Phl. ?, NP. $\sqrt{ } z \bar{a} r$.

5. Kj. $z=$ Av. $c h$, Phl. $j$, NP. $z$.

$\sqrt{ }$ pārēz, abstain, protect, defend: Av. paiti- $\sqrt{ }$ raech, Phl. $\checkmark$ pahrēj, NP. parhīz.

6. Kj. $z=$ Av. $t$, Phl. $s h$, NP. $s h$.

khī, relatives: Av. khvaetu-, Phl., NP. khvessh.

7. Kj. $z=$ Av. $s$, Phl. $s$, NP. $s$.

zīw, silver: Av. simā, Phl. asīm, NP. sìm. nizm, low : Av. nisma, Phl., NP. - quz, uterus : Av. ?, Phl. ?, NP. kus.

8. Kj. $z=$ Av. ?, Phl. sh, NP. sh. zik, belly: Av. ?, Phl. shikambu, NP. shikam.

9. Kj. $z=$ Ar. $\dot{~} \dot{~}$.

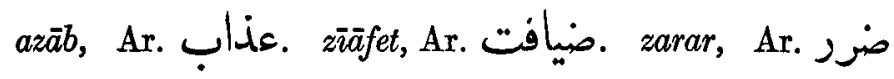

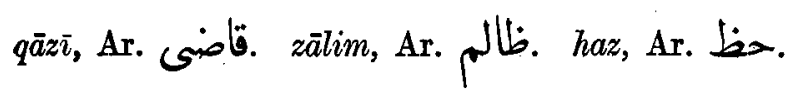


XXXVII. $z h$

1. Kj. $z h=$ Av. $z h$, Phl. $z, j$, NP. $z$.

$z h n \bar{u}, a z h n \bar{u}, \mathrm{knee}:$ Av. $z h n \bar{u}-$, Phl., NP. $z \bar{a} n \bar{u}=$ Av. $z \bar{a} n \bar{u}$. tūzh, sharp: Av. taezha-, tizhi-, Phl. tēj, NP. tēz.

2. $\mathrm{Kj} . z h=\mathrm{A} \nabla . z$, Phl. $z, \mathrm{NP} . z$.

$\bar{a} z h \bar{a} r$, torment: Av. $\bar{a}-\sqrt{ } z a r$, Phl., NP. $\bar{a} z \bar{a} r . \sqrt{ } \bar{a} z h \bar{u}$, urge, drive: Av. $\bar{a}-\sqrt{ } a z$, Phl., NP. -

3. $\mathrm{Kj} . z h=\mathrm{Av} . z$, Phl. $z h, \mathrm{NP} . z$. hazhār, wretched, miserable: Av. $\sqrt{ } z a r$, Phl. $z h \bar{a} r$, NP. $z a \bar{r}$.

4. Kj. $z h=$ Av. j, Phl. $j$, NP. 一. $h \bar{a} z h$, strength : Av. aoja-, Phl. ōj, NP. 一.

5. Kj. $z h=$ Av. j, Phl. $z$, NP. $z h$. zhür, chamber, cellar : Av. jafra-, Phl. zufar, NP. zharf.

6. Kj. $z h=$ Av.j, Phl. $z$, NP. $z$.

zhin, woman: Av. jēn̄-, Phl., NP. zan. $\sqrt{ } z h \bar{\imath}$, live: Av. $\sqrt{ } j i$, Phl. $\sqrt{ } z i v$, NP. $\sqrt{ } z i . \quad \sqrt{ } z h a n$, churn, beat: Av. $\sqrt{ } j a n$, Phl., NP. $\sqrt{ } z a n$. zhār, poison; Av. jatra, Phl., NP. zahr.

7. $\mathrm{Kj} . z h=\mathrm{Av}$. $c h$, Phl. $c h, \mathrm{NP} . z$. $\sqrt{ }$ rēzh, pour: Av. $\sqrt{ }$ rech, Phl. $\sqrt{ }$ rech, NP. $\sqrt{ }$ rizz. $r \bar{o} z h$, light, day: Av. raochah-, Phl. rōch, NP. rūz.

8. $\mathrm{Kj} . z h=c h$ in loan-word.

halōzha, plum : NP. älücheh.

9. Kj. $z h=$ Av. $s$, Phl. $s$, NP. $s$.

qizh, locks : Av. gaesa-, Phl., NP. gès.

10. Kj. $z h=$ Av. $s$, Phl. sh, NP. sh. jēzhn, festival : Av. yasna, Phl. yashn, NP. jashn.

11. Kj. $z h=$ Av. $s h$, Phl. $s, s h$, NP. $s, s h$.

$\sqrt{ }$ chezzh, taste: Av. $\sqrt{ }$ chash, Phl., NP. $\sqrt{ }$ chash. $\sqrt{ } k u z h$,

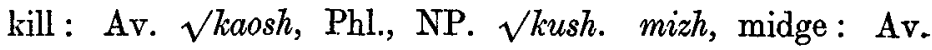
makhshi-, Phl., NP. magas.

12. Kj. $z h==$ Av. $d$, Phl. $z$, NP. $z h$.

zhuzhik, hedgehog : Av. duzhaka-, Phl. zūzak, NP. zhūzhī. 


\section{Aspirate \\ XXXVIII. $h$}

Occurs only initially, not having survived medially or finally.

1. $\mathrm{Kj} . h=\mathrm{Av} . h$, Phl. $h, \mathrm{NP} . h$.

hāwan, mortar: Av. hāvana-, Phl., NP. hāvan. hāwīn, summer: Av. hāmin, Phl. hāmīn, NP. 一.

2. $\mathrm{Kj} . h=$ Av. $h$, Phl. - NP. - .

hagar, if: Av. ha-kara-, Phl., NP. agar. han-, verbal prefix: Av. ham-, Phl., NP. an-. hì, genitive particle: Av. hē, OP. hya, Phl. i, NP. $i$. (the $i z \bar{a} f a)$.

3. $\mathrm{Kj} . h=$ Phl. $h$, NP. - .

harmī, pear: Phl. hormod, NP. armüd.

4. $\mathrm{Kj} . h=$ Av. $h$, Phl. $k h$, NP. $k h$.

hishk, dry: Av. hushka-, Phl., NP. khushk. hu, hog: Av. $h u^{-}$, Phl., NP. $k h \bar{u} k$.

5. Kj. $h=$ Av. -, Phl. $k h$, NP. $k h$.

hilka, hēe, egg: Av. ?, Phl. khāik, NP. khāya. hïrch, bear: Av. aresha-, Phl., NP. khirs.

6. $\mathrm{Kj} . h=\mathrm{NP}$. $k h$.

hēnik, cool: NP. khunūk.

7. $\mathrm{Kj} . h=k h$ in loan-word.

hawīr, dough : Ar.

8. $\mathrm{Kj} . h=y$ in loan-words.

hatīw, orphan: Ar. ث̈... hēldāsh, travelling companion : Tk. yoldāsh. hêes̄sh, gently: Tk. yawāsh.

9. $\mathrm{Kj} . h=\mathrm{Kj} . w$.

hirch $=$ wirch. $\quad$ hishk $=$ wishk. $\quad$ henāsa $=$ wenāsa. $\quad$ hurd $=$ wurd.

10. $\mathrm{Kj} . h$ occurs as augment before $a$ or $\bar{a}$.

(a) In the following original words it is an augment:

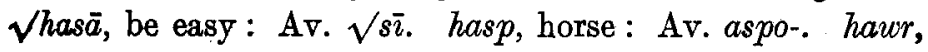


cloud: Av. awra-. hawrāz, ascent: Av. aiwi-rāsta. $\sqrt{ } h \bar{a}$, come: Av. $\sqrt{ } \bar{a} i$. hāzh, power: Av. aoja-. $\sqrt{ } h \bar{a} n$, bring: Av. $\bar{a}-\sqrt{ }$ nay.

(b) In the following loan-words: halözha: NP. alücha.

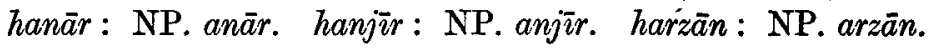
hasīr: Ar. hājiz: Ar. اسير. halak: Tk. alak; and many others.

(c) In hastūr, Av. stawra-, Phl. stavr, NP. sitabr; and in hawär (camping ground), Av. vara-, Phl. var, NP. - , it appears with $-a$, as $h a$ augment.

\section{XXXIX. $\underline{h}$}

The guttural aspirate, which is not natural to $\mathrm{Kj}$., being borrowed from Arabic. It is not always preserved in loanwords from Arabic, tending to become a simple aspirate, as in haz, Ar. حمظ ; حفر ; hafr, Ar. Ar. حطاضر.

In one case only has $h$ become $h$, namely in hawt, seven : Av. hapta-, which is, however, only thus pronounced in a small area in the south.

\section{LOSSES OF ORIGINAL LETTERS AND TREATMENT OF SOME CONSONANT GROUPS}

1. The commonest loss is that of original $d$ and $t$, which is supported by hundreds of examples both ancient and modern. The dental is, however, preserved in the initial position. After a long vowel, between vowels, and after $n$, it invariably disappears.

In all the following examples both $\mathrm{Phl}$. and NP. preserve the original $t$, the former as $t$ or $d$, the latter as $d$.

ganum, wheat: Av. gantaoma-. wā, wind: Av. vāta-. gan, fetid: Av. gainti-. $\sqrt{ }$ gar, turn: Av. $\sqrt{ }$ veret. kām, which : Ab. katama-. $\bar{a} z \bar{a}$, brave; Av. $\bar{a} z \bar{a} t a-. \quad b \bar{\imath}$, willow : Av. vaeiti-. spi, good, pretty, white: Av. spaeta-, etc.

2. Original $t h r$ preceded by a short vowel becomes $\bar{a} r$, while 
in Phl. and NP. original short vowel is preserved and thr changed to $t r$ or $h r$.

chār, face : Av. chithra-, Phl. chihr, NP. chihra.

zhār, poison : Av. jathra-, Phl., NP. zahr.

shār, district, township : Av. khshathra-, Phl. shatr, NP. shahr.

The group èra, awra, and küra, seen in their proper forms in Central and Northern Kurdistan as $\bar{\imath} d e r$, avder, and küder (Kj. koñder), suggest comparison with Av. ithra, avathra, and kuthra, with simple loss of $t h$, as in chwār, where the $w$ of Av. chathwār, is preserved and $t h$ lost, as against the reverse process in Phl. and NP., where the th has been preserved in the $h$ of chahār, and no trace of $w$ survives.

3. Original medial $k h r$ only appears to be represented in the word sür, red ; Av. sukhra. Phl. has preserved the group in sukhr, while NP. has inverted it in surkh. From the presence of suhr in Central and Northern Kj., it would appear the $s \bar{u} r$ is the result of loss of $k h$ before $r$ with the vowel lengthening noticeable with the loss of original th before $r$.

Original medial $f r$ is preserved intact in one case, and as $\bar{u} r$ in another, without inversion as in $\mathrm{Phl}$. and NP.

wafr, snow : Av. vafra-, Phl. vafr, varf, NP. barf.

zhür, chamber, cellar : Av. jafra-, Phl. zafr, NP. zharf.

In the second example of appears to have passed through aw to $\vec{u}$. The form zhaur is heard in the extreme south.

4. Original $m r$ only appears in nerm, Av. namra, Phl., NP. narm, which may be a loan-word from Phl. or NP.

5. Original $s r$ appears in asr, tears, Av. asru-, Phl., NP. ars, i.e. not inverted, as in Phl. and NP.

6. The word chawr, fat, grease $=$ Phl. charp, NP. charb, indicates the preservation of a possible $b r, p r$ group.

7. Original consonant(s) plus $n$. This may be subdivided into $k h s h n$, other consonant plus $n$, and group $r-n$.

In the first case $\mathrm{Kj}$. loses $k h s h$, while $\mathrm{Phl}$. and NP. preserve sh, losing $k h$. 
$\sqrt{ } n \bar{a} s$, know, recognize: Av. $\sqrt{ } k h s h n a \bar{s}$, Phl. $\sqrt{ } s h n \bar{a} s$, NP. $\sqrt{ }$ shinās.

rün, light: Av. raokhshna = Phl., NP. rōshan.

In the second case $\mathrm{Kj}$. loses any consonant preceding $n$, while Phl. and NP. preserve it.

rūn, oil : Av. raoghna-, Phl., NP. röghan.

tìn, thirst : Av. tarshna, Phl., NP. tashna.

pāni, heel: Av. pāshna, Phl., NP. pāshna.

The treatment of original $r-n$ is the same as Phl. and NP. viz. loss of $n$, as par : Av. parena-, etc.

8. Treatment of original $k h$.

As an initial, before sh, Kj., like Phl. and NP., drops $k h$, as in shaw, shìr, etc., from Av. khshapa-, khshīra-, Phl. shap, shìr, NP. shab, shīr.

As an initial, where $\mathrm{Phl}$. and NP. preserve $k h, \mathrm{Kj}$. loses it, as :-

wash, pleasant: Av. ?, Phl. khvash, NP. khüsh. wurd, small : Av. $\sqrt{ }$ khvar, Phl. khurtak, NP. khurd. shìn, blue, green: Av. khshaena-, Phl. khashīn, NP. khashīn.

As a medial, where $\mathrm{Phl}$. and NP. preserve $k h, \mathrm{Kj}$. loses it, as :-

bash, part, lot: Av. bakhsh, Phl., NP. bakhsh.

mēsh, fly: Av. makhshi-, (Phl., NP. magas).

tūm, seeds : Av. taokhma-, Phl. tokhm, NP. tukhm.

wach, twig: Av. vakhsha-, Phl. vakhsh, NP. 一.

9. $\mathrm{Kj}$. loses original $s h$ where it is the final consonant, while Phl. and NP. preserve it.

ispī, louse : Av. spish-, Phl. shupush, NP. shepish.

gұ̨e, ear : Av. gaosha-, Phl., NP. gösh.

mè, ewe: Av. mazsha-, Phl., NP. mësh.

rëwī, fox: Av. revish-, Phl. rōbās, NP. rūbāh.

rē, beard: Av. razsha-, Phl. rḕsh, NP. rīsh. 
10. In the word sipul, $\mathrm{Kj}$. has lost the $z$ of Av. spereza-, which is preserved in Phl. and NP. in sparz and sipurz. In brīka. "flashing, sparks," a similar loss of $z$ (Av. $\sqrt{ }$ braz) is noticed, though it is preserved as $s$ in briska, having the same meaning.

11. Kj. loses final $c h$, preserved by $\mathrm{Phl}$. and changing to $z$ in NP.

rō, light, day : Av. raochah-, Phl. roch, NP. rūz.

$\sqrt{ }$ tw, flow, melt: Av. $\sqrt{ }$ tach, Phl. $\sqrt{ }$ vitāch, NP. $\sqrt{ }$ gudāz.

12. Loss of $r$.

? initial (unsupported by other examples) :-

äst, direction, line: Av. rāst-, Phl., NP. rast.

Medial :-

tīn, thirst: Av. tarshna-, Phl. tishn, NP. tishna.

$\bar{e} z h$, worth : Av. arejah-, Phl. arj, NP. arz.

anishk, elbow : Av. arethn-, Phl. aret, ertan, NP. āranj.

baosh, upper part of side : Av. barozhda-, Phl., NP. -

$\sqrt{b} a$, take, bear: Av. $\sqrt{ }$ bere, Phl., NP. $\sqrt{ } b a r$.

Final :-

khasū, mother-in-law : Av. khvasüra, Phl. ?, NP. khasūr. $\sqrt{ } \bar{a} z h i$, flood, inundate: Av. $\bar{a}-\sqrt{ }$ ghzhar, Phl., NP. - . $\sqrt{ }$ pzha, sprinkle continuously: Av. vi- $\sqrt{g h z h a r, ~ P h l ., ~}$ NP. - .

13. Loss of $t$ preserved in Phl.

$\sqrt{ } g \bar{u} r$, change, return: Av. $\sqrt{ }$ varet, Phl. $\sqrt{ }$ vart, NP. $\sqrt{ } g \bar{a} r$.

bist, span : Av. vi-tasti-, Phl. vitast, NP. bidhist.

14. Loss of medial $b$ :-

tūr, radish : NP. turb-izza.

tūra, wallet, loan-word from Tk. tubra.

Loss of $b$ after $m:-$

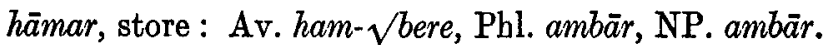

shamu, Sabbath : NP. shambeh.

gumez, dome: NP. gumbad.

JRAS. APRIL 1922. 
15. Loss of final $v$.

$$
g \bar{a}, \text { bull : Av. gā v-, Phl., NP. gāv. }
$$

16. Loss of $g h$ preserved in $\mathrm{Phl}$. and NP.

drū, a lie: Av. draogha-, Phl. drogh, NP. durōgh.

rün, butter: Av. raoghna-, Phl. rokan, NP. röghan.

mir-ishk, hen : Av. meregha-, Phl., NP. murgh.

Authorities consulted : Grundriss der Iranischen Philologie; Grundriss der Neupersischen Etymologie, Horn; Kurdische Grammatik, Justi ; Dictionnaire Kurde Francaise, JustiJaba ; An old Zand Pahlavi Glossary, Jamaspji; AvestaEnglish and English-Avesta Glossary, Bharucha; An Avesta Grammar, Williams Jackson; Études Iraniennes, Darmesteter. 